\title{
Prevalencia de Caries en Alumnos de Educación Básica y su Asociación con el Estado Nutricional
}

\author{
MARÍA ANGÉLICA CERECEDA M. ${ }^{1}$, SIMONE FALEIROS C. ${ }^{2}$, ANDREA ORMEÑO Q. ${ }^{3}$, \\ MAYERLING PINTO G. ${ }^{4}$, REBECA TAPIA V. ${ }^{5}$, CARLOS DÍAZ S. ${ }^{6}$, HERNÁN GARCÍA B. ${ }^{7}$
}

1. Odontopediatra. Profesora Asistente, Asignatura Odontopediatría, Facultad de Odontología, Universidad de Chile.

2. Odontóloga. Becaria CONICYT, Programa de Doctorado en Salud Pública, Facultad de Medicina, Universidad de Chile.

3. Docente Asignatura Odontopediatría, Facultad de Odontología, Universidad de los Andes.

4. Docente Asignatura Odontopediatría, Facultad de Odontología, Universidad de Chile y Universidad de los Andes.

5. Docente Asignatura Odontopediatría, Facultad de Odontología, Universidad de los Andes.

6. Médico, Epidemiólogo Clínico. Becario Proyecto MECESUP, Programa de Doctorado en Salud Pública, Facultad de Medicina, Universidad de Chile.

7. Médico Pediatra. Endocrinólogo Infantil. Docente Asignatura de Pediatría, Facultad de Medicina, Pontificia Universidad Católica de Chile.

\section{ABSTRACT \\ Prevalence of Dental Decay in Elementary School Children and Association with Nutritional Status}

Introduction: It is thought that obesity is a risk factor in the development of dental decay in children. However there is no agreement in the literature in this area. Objective: To define the prevalence of caries among lower class school children in the metropolitan region in Santiago, Chile, years 2006-2007, and observe its association with the children's nutritional status. Subjects and Methods: A random sample of children 5-15 years of age, in 8 primary schools provided a stratified population of 1190 individuals. Oral examination provided a health index, nutritional status was determined using CDC 2000. Statistical analyses were performed utilizing Stata 9,0. Results: Prevalence of dental caries in the total population was 79,5\%. Prevalence of decay among eutrophic, overweight and obese children was $80,0 \%, 78,1 \%$ y $79,9 \%$ respectively. Conclusion: This population presents a very high prevalence of dental caries, especially among a group classified a"normal". In this sample, there was no statistically significant association betweeen dental decay and nutritional status.

(Key words: Decay, nutritonial status, association).

Rev Chil Pediatr 2010; 81 (1): 28-36

\section{RESUMEN}

Introducción: Se ha postulado que la obesidad constituiría un factor de riesgo para el desarrollo de caries en niños, sin embargo, la literatura publicada muestra información discordante respecto de esta asociación. Objetivo: Conocer la prevalencia de caries en escolares de clase media baja de región metropolitana de San-

Trabajo recibido el 14 de septiembre de 2009, devuelto para corregir el 05 de octubre de 2009, segunda versión el 01 de diciembre de 2009, aceptado para publicación el 17 de diciembre de 2009.

Correspondencia a:

M. Angélica Cereceda

E-mail: macereceda@gmail.com 
tiago, Chile y determinar su asociación con el estado nutricional de los mismos, entre los años 2006 y 2007. Materiales y Métodos: Se ejecutó un estudio de corte transversal en 8 colegios de la Sociedad de Instrucción Primaria (SIP). Se seleccionó, mediante un muestreo aleatorio estratificado por género y curso, una muestra de 1190 escolares de 5 a 15 años. Mediante examen bucal se consignó índice COPD. Se determinó el estado nutricional mediante la utilización de la referencia CDC 2000. Para los cálculos y estimaciones estadísticas se utilizó el programa Stata 9,0. Resultados: La prevalencia de caries en la población total fue de 79,5\%. La prevalencia de caries en los niños eutróficos, con sobrepeso y obesos fue de $80,0 \%, 78,1 \%$ y $79,9 \%$ respectivamente. Conclusión: La población evaluada presenta una alta prevalencia de caries, sobretodo en el grupo clasificado como normal. En esta muestra no se encontró una asociación estadísticamente significativa entre la prevalencia de caries y el estado nutricional.

(Palabras clave: Caries, estado nutricional, asociación).

Rev Chil Pediatr 2010; 81 (1): 28-36

\section{Introducción}

La obesidad y el sobrepeso han mostrado un aumento importante en los últimos años en la población mundial. En lo que concierne a la población infantil y adolescente, este incremento ha sido descrito como una gran preocupación en Salud Pública ${ }^{1}$.

En Chile, la observación de la tendencia de mal nutrición por exceso en diferentes estudios transversales realizados en varias localidades a lo largo del país, demuestra que entre los años 1986 y 1998, se registró un importante incremento del sobrepeso infantil: de 8,3\% a 19,6\% en niños prepúberes, y de $10,2 \%$ a $16,2 \%$ en niñas prepúberes. Por otro lado, la obesidad también aumentó de manera significativa: de $4,3 \%$ a $29,5 \%$ en niños prepúberes y de $4,7 \%$ a $24 \%$ en niñas prepúberes ${ }^{2}$. Según datos de la Junta Nacional de Auxilio Escolar y Becas (JUNAEB), la prevalencia de obesidad en escolares de primer año básico continua siendo alta $^{3}$.

Por otro lado, la caries dental constituye una de las enfermedades más importantes de la odontología y, en la infancia, representa un importante desafío para la Salud Pública. La Organización Mundial de la Salud ha estimado que entre el 60 y $90 \%$ de los niños del mundo presentan lesiones de caries con cavitación evidente ${ }^{4}$. En Chile, los últimos estudios de prevalencia realizados por el Ministerio de Salud, mostraron la presencia de altos índices de caries en la población infantil, con una prevalencia, en niños (as) de 6 y 12 años, de 70,3\% y $62,5 \%$, respectivamente ${ }^{5,6}$.
Se ha enunciado que la obesidad constituiría un factor de riesgo para el desarrollo de caries, sin embargo, la literatura publicada muestra información discordante respecto de esta asociación ${ }^{7-9}$. Teóricamente, la mal nutrición por exceso debiera estar asociada a la caries dental, dado que ambos, en principio, son causados por los mismos factores. Existe evidencia que avala la asociación entre caries y hábitos alimentares caracterizados por consumo de azúcares y carbohidratos refinados, especialmente si este es frecuente ${ }^{10}$. Frente a esto, se podría considerar que la mal nutrición por exceso debiera también representar un marcador para la experiencia de caries en niños y adolescentes ${ }^{11}$.

Pocos estudios acerca de la asociación entre caries dental y mal nutrición por exceso han sido publicados hasta el momento. En Chile, no existen estudios que determinen la carga de esta enfermedad en la población infantil y su posible asociación con el estado nutricional.

El objetivo del presente estudio es conocer la prevalencia de caries en los escolares de nivel socioeconómico medio-bajo y bajo, que asisten a Escuelas de la Sociedad de Instrucción Primaria de Santiago (SIP), distribuidos en diferentes comunas de Santiago, y determinar la asociación existente entre la experiencia de caries y el estado nutricional, en el año 2006-2007.

\section{Materiales y Métodos}

Considerando el objetivo de este estudio, se diseñó un estudio descriptivo, observacional, 
de corte transversal. La población a estudiar está constituida por escolares de primero a octavo básico, de ambos sexos, y pertenecientes a comunidades de ingreso socioeconómico medio-bajo y bajo. Para ello se utilizaron los alumnos de educación básica de los colegios de la Sociedad de Instrucción Primaria de Santiago-SIP (Colegios Matte). Esta institución cuenta con 15 establecimientos de educación básica. Estos planteles se encuentran distribuidos en distintas comunas de la ciudad de Santiago, excepto en el sector oriente de la capital, y cuentan con una población escolar total aproximada de 15000 alumnos de educación básica. Con el objetivo de obtener una muestra representativa de la SIP, la selección de los sujetos se realizó mediante un muestreo aleatorio multietápico y por conglomerados. Los planteles de la SIP corresponden a colegios particulares subvencionados orientados a la clase media-baja y baja de la comunidad. Se seleccionó, mediante sorteo aleatorio, a 8 de los 15 colegios, los cuales quedaron distribuidos en 7 comunas de la capital. Dentro de cada establecimiento, se realizó un muestreo aleatorio estratificado por sexo y curso, seleccionándose a aproximadamente 6 niños ( 3 hombres y 3 mujeres) dentro de cada curso (alrededor de 24 cursos en cada colegio). Para la generación de los números aleatorios, se utilizó el servicio denominado "True Random Number Service" mantenido por el Colegio de Estadísticos y Ciencias Computacionales del "Trinity Colle$g e$ " en Dublin, Irlanda ${ }^{12}$.

Para fines de tamaño muestral, el nivel de confianza del presente estudio es de 95\% ( $Z$ 1 -alfa $=1,96)$ y se consideró un error de estimación de $3 \%$. Al considerar el peor escenario posible para el cálculo de tamaño muestral, con una prevalencia de caries de un $50 \%$, se requiere de un mínimo de 1050 alumnos para determinar la prevalencia de caries en la población del estudio. La muestra final fue constituida por 1190 alumnos, siendo un promedio de 149 alumnos por colegio y de 18 alumnos por nivel, seleccionados mediante sorteo aleatorio. Respecto al cálculo del tamaño muestral para el estudio de asociación (Odds Ratio), se consideró además, un poder de $80 \%$, esperándose encontrar una diferencia de al menos $10 \%$ en la experiencia de caries entre niños con mal nutrición por exceso y niños eutróficos. En tal escenario, se requieren 288 niños en cada grupo. La población de niños con mal nutrición por exceso en el presente estudio fue de 561 y de niños normales fue de 612.

Se incluyó en el estudio los niños cuyos padres hayan aceptado su participación mediante consentimiento informado. Además los escolares debieron dar su asentimiento para participar.

Para la medición del peso corporal el niño debió estar descalzo en ambiente temperado, con un mínimo de ropa. Se realizaron tres mediciones en pesa marca SECA modelo 700, previamente calibrada mediante la utilización de pesos fijos conocidos, determinándose el peso en kilos con un decimal. El resultado final se consignó como el promedio de las mediciones. Todos los niños de la muestra fueron pesados durante el período matinal, entre las ocho y las doce horas.

La talla fue determinada con el niño en posición erecta, empleando un estadiómetro de pared fijo marca SECA previamente calibrado. Se codificó en centímetros más un decimal (ej. $100,5=$ cien centímetros con 5 milímetros). Fueron realizadas tres mediciones y el resultado final se consignó como el promedio de éstas.

Las medidas antropométricas fueron realizadas por un equipo de 8 pediatras y 16 internos de medicina previamente capacitados. El sobrepeso y la obesidad se definieron mediante el índice de masa corporal (IMC) tomándose el peso del niño y dividiéndose por el cuadrado de su talla. Los valores obtenidos de IMC se compararon con las tablas estándares del Centro Nacional para Estadísticas de Salud de Estados Unidos de Norteamérica (CDC-NCHS, National Center for Health Statistics) según la edad y el sexo del alumno ${ }^{13}$.

Los alumnos de bajo peso son los que presentaron índice de masa corporal con percentil menor o igual a 5. Se definió como sobrepeso el IMC mayor o igual al percentil 85 y menor que el percentil 95, y obesidad cuando este fue mayor o igual al percentil 95, según edad y sexo. Se consideraron eutróficos aquellos alumnos que presentaron IMC con percentil mayor que 5 y menor que 85 . El término mal nutrición por exceso se refiere al grupo de ni- 
ños que presentan sobrepeso y obesidad.

La caries dental en el presente trabajo fue considerada como lesión con cavidad evidente, detectable mediante examen visual. La experiencia de caries fue medida a través de los índices COPD y CEOD, los cuales describen, respectivamente, la prevalencia de caries en dentición permanente y temporal en un individuo, mediante el número de piezas cariadas, obturadas y perdidas o con extracción indicada por motivo de caries. Finalmente, se midió el promedio comunitario de estos índices como indicadores de experiencia de caries de la población total del estudio.

El examen bucal fue realizado por un equipo de 4 odontólogos previamente estandarizados en lo referente al diagnóstico de caries, registrándose la información en una ficha clínica. La capacitación se realizó mediante la visualización de 20 imágenes computadorizadas de todos los puntos considerados a partir de las recomendaciones de la Organización Mundial de la Salud (OMS) para el examen clínico, y luego con diferentes casos clínicos. Se realizaron 4 sesiones de debate en conjunto en distintos días, bajo las mismas condiciones de luz, y una sesión de examen final de las imágenes. Posteriormente, los cuatro examinadores revisaron a 10 pacientes cada uno, siguiendo las mismas recomendaciones ${ }^{14}$.

Se siguió los parámetros de diagnóstico recomendados por la $\mathrm{OMS}^{15}$. Los niños fueron examinados estando sentados en una silla, frente a una ventana (buena iluminación) y se realizó inspección visual de los tejidos blandos y duros de la cavidad bucal. Como instrumento táctil se utilizó una sonda recta y una sonda periodontal en los niños que presentaron dentición permanente, así como espejo dental $\mathrm{N}^{\mathrm{o}} 5$ y una linterna para visualización de áreas de difícil acceso.

El presente trabajo fue presentado y aprobado para su realización, por el Comité de Ética de la Investigación en Seres Humanos de la Facultad de Medicina de la Universidad de Chile. Se obtuvo el consentimiento informado de los padres o apoderados de los niños que participaron en el estudio. Como beneficio a los participantes, se procedió a consejería en dieta, salud e higiene oral a los niños. No fue realizada ninguna maniobra que pudiera representar riesgo o daño al participante durante la obtención de los datos.

\section{Resultados}

El número total de alumnos examinados fue de 1190 , con una proporción levemente superior de mujeres $(51,6 \%)$. La diferencia de frecuencia entre los grupos no fue significativa $(\mathrm{p}=0,09)$. El promedio de edad de la población fue de 9,7 años y el rango de edad varió desde los 5 a los 15 años (tabla 1).

La diferencia entre la proporción de niñas en la presente muestra y aquella encontrada en la población general, estimada por el Instituto Nacional de Estadísticas de Chile ${ }^{16}$, no fue significativa $(p=0,19)$. Situación semejante se observó en la población de varones $(\mathrm{p}=0,21)$.

La prevalencia de sobrepeso en la población total fue de 25\% (IC95\% 22,5 - 27,5), y de obesidad fue de 22\% (IC95\% 19,8 -24,6). La distribución de la muestra según estado nutricional y sexo se presenta en la tabla 2.

La prevalencia total de caries, considerando piezas temporales y definitivas de toda la

Tabla 1. Distribución de la muestra según edad y sexo

\begin{tabular}{|c|c|c|c|c|c|c|}
\hline \multirow[t]{3}{*}{ Edad } & \multicolumn{4}{|c|}{ Sexo } & \multicolumn{2}{|c|}{ Total } \\
\hline & \multicolumn{2}{|c|}{ Hombres } & \multicolumn{2}{|c|}{ Mujeres } & \multirow[b]{2}{*}{$n$} & \multirow[b]{2}{*}{$\%$} \\
\hline & n & $\%$ & $\mathbf{n}$ & $\%$ & & \\
\hline $5-8$ & 156 & 13,1 & 222 & 18,6 & 378 & 31,7 \\
\hline $9-11$ & 254 & 21,3 & 252 & 21,1 & 506 & 42,5 \\
\hline $12-15$ & 164 & 13,7 & 142 & 11,9 & 306 & 25,7 \\
\hline Total & 574 & 48,2 & 616 & 51,6 & 1190 & 100,0 \\
\hline
\end{tabular}


Tabla 2. Distribución de la muestra según estado nutricional y sexo

\begin{tabular}{|c|c|c|c|c|c|c|}
\hline \multirow[t]{3}{*}{ Estado Nutricional } & \multicolumn{4}{|c|}{ Sexo } & \multicolumn{2}{|c|}{ Total } \\
\hline & \multicolumn{2}{|c|}{ Hombres } & \multicolumn{2}{|c|}{ Mujeres } & \multirow[b]{2}{*}{ n } & \multirow[b]{2}{*}{$\%$} \\
\hline & $n$ & $\%$ & n & $\%$ & & \\
\hline Bajo Peso & 8 & 53,3 & 7 & 46,6 & 15 & 1,2 \\
\hline Normal & 291 & 47,3 & 323 & 52,6 & 614 & 51,6 \\
\hline Sobrepeso & 140 & 47,1 & 157 & 52,8 & 297 & 24,9 \\
\hline Obesidad & 135 & 51,1 & 129 & 48,8 & 264 & 22,1 \\
\hline Total & 574 & 48,2 & 616 & 51,7 & 1190 & 100,0 \\
\hline
\end{tabular}

población fue de 79,5\% (IC95\% 77,1-81,7). La prevalencia de caries en piezas definitivas fue de 59,4\% (IC95\% 56,6-62,2). El promedio de caries en piezas temporales (ceod) fue de 2,7 (IC95\% 2,5-2,8) y en piezas permanentes (COPD) fue de 1,8 (IC95\% 1,7-2,0). A la edad de 12 años, el COPD fue de 2,8 (IC95\% 2,4$3,2)$. La prevalencia total de caries por estado nutricional es presentada en la figura 1 .

El detalle de esta información se presenta en la figura 2, donde se observa que, aunque la prevalencia de caries es aparentemente mayor en los hombres en comparación con las mujeres, tal diferencia no es estadísticamente significativa $(\mathrm{p}=0,39)$. Asimismo, la prevalencia de caries en niños diagnosticados con mal nutrición por exceso no mostró ser significativamente distinta a la de los niños considerados normales $(p=0,27)$.

Como forma de controlar el efecto confundente de las variables sexo y edad, y para buscar asociación entre sobrepeso y obesidad sobre la experiencia de caries, se procedió al análisis de regresión logística, encontrándose que el estado nutricional no se relaciona con la experiencia global de caries en la presente población de estudio (tabla 3).

Con el objetivo de considerar el efecto que pueda ejercer el establecimiento educacional sobre la experiencia de caries en los alumnos, se realizó un análisis multinivel, considerando el colegio como macro nivel (o nivel estructural) y el niño como micro nivel (o nivel individual). Tomando en cuenta dicho contexto, se procedió al análisis de regresión lineal múltiple, donde se evaluó el efecto de la variabilidad en el índice IMC sobre la prevalencia de caries

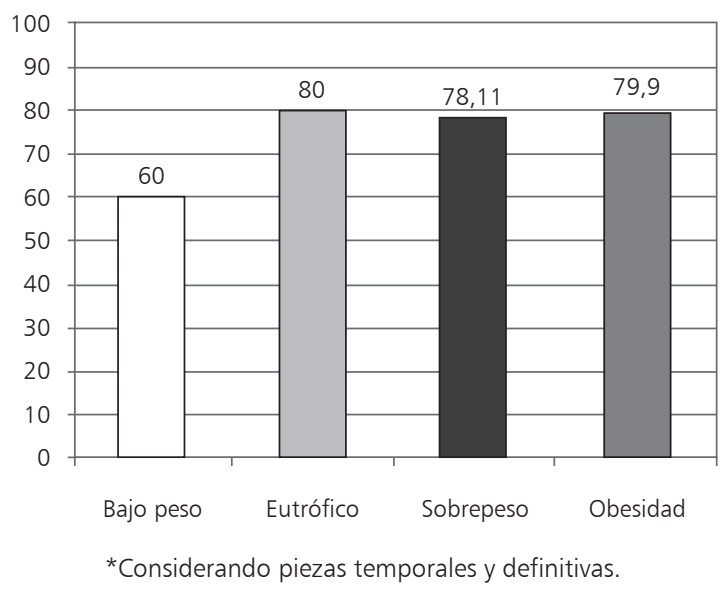

Figura 1. Prevalencia total de caries (\%) según estado nutricional.

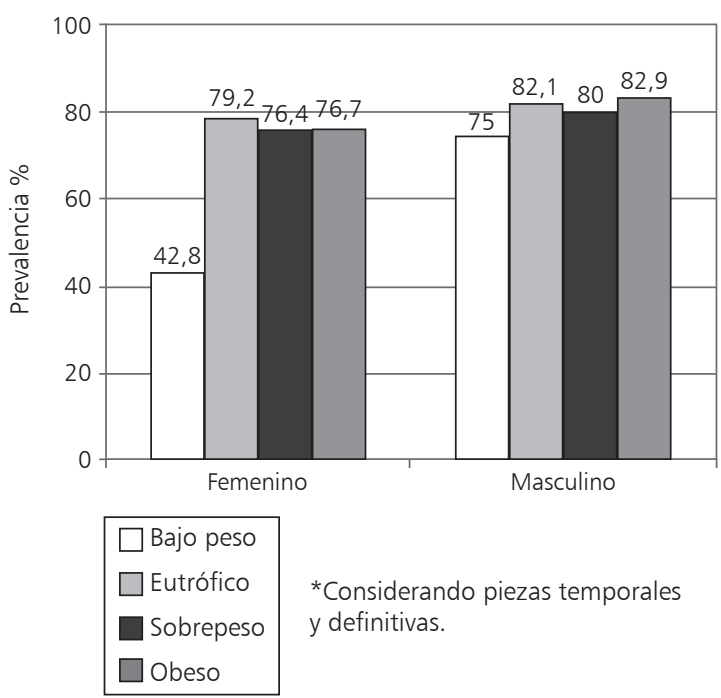

Figura 2. Prevalencia de caries (\%) según estado nutricional y sexo. 
Tabla 3. Modelo* de Asociación Entre Estado Nutricional y Caries Dentales ${ }^{1}$

\begin{tabular}{|lcccc|}
\hline Estado Nutricional & OR Crudo & IC $\mathbf{9 5}^{\mathbf{3}}$ & OR Ajustado & IC 95\% $^{\mathbf{3}}$ \\
\hline Bajo Peso $^{2}$ & 0,36 & $(0,12-1,03)$ & 0,35 & $(0,12-1,01)$ \\
\hline Sobrepeso $^{2}$ & 0,86 & $(0,61-1,21)$ & 0,86 & $(0,61-1,21)$ \\
\hline Obesidad $^{2}$ & 0,96 & $(0,66-1,37)$ & 0,96 & $(0,66-1,38)$ \\
\hline
\end{tabular}

*Regresión Logística. 'Dicotomizada en "libre de caries" o "presencia de una o más lesiones de caries". ${ }^{2}$ Valor de referencia: Estado Nutricional Eutrófico o Normal. ${ }^{3}$ Sin significancia estadística $(p>0,05) .{ }^{4}$ Odds ratio ajustado según sexo y edad.

en los alumnos, ajustado por edad y sexo, para cada modalidad de dentición, no encontrándose asociación significativa en ninguno de los casos (dentición permanente: $\mathrm{p}=0,42$; dentición temporal: $\mathrm{p}=0,56 \mathrm{y}$ dentición mixta: $\mathrm{p}=0,51)$. La variabilidad explicada por el nivel superior (variabilidad entre colegios) fue de $2,6 \%$ (IC95\% 1,3-4,9) en lo que concierne al total de caries.

\section{Discusión}

El presente estudio tuvo por objetivo conocer la prevalencia de caries en escolares de nivel socioeconómico medio bajo y bajo, alumnos de la Sociedad de Instrucción Primaria de Santiago de Chile (SIP), y determinar la asociación existente entre la experiencia de caries y el estado nutricional. Para la muestra estudiada, no se encontró evidencia de asociación entre las variables mencionadas.

El índice COPD a los 12 años es un indicador de la salud oral ampliamente utilizado a nivel internacional. En esta población este indicador fue de 2,8, situándose por encima del valor nacional de $1,9^{5,6}$. Una revisión realizada por la Organización Panamericana de la Salud (OPS) clasifica a los países según este indicador en tres categorías: menor de 3 , entre 3 y 5 , y mayor de $5^{17}$. Si se compara el valor del COPD de la presente muestra con el obtenido en otros países americanos, esta población se encontraría en el rango menor de 3 , similar a la de la mayoría de los países actualmente.

La investigación de la asociación entre la experiencia de caries y el estado nutricional en niños no ha sido muy explorada. La literatura existente es escasa y no concluyente en lo referente a los resultados encontrados ${ }^{7}$. El presente estudio no encontró asociación entre la experiencia de caries y la mal nutrición por exceso (sobrepeso y obesidad) en los niños de la muestra. De la misma forma, no se encontró evidencia para afirmar que el incremento en el índice de masa corporal se asocia a una mayor severidad de la experiencia de caries.

Tales hallazgos son coincidentes con los de Pinto y $\mathrm{col}^{9}$, que, en el año 2007, realizaron un estudio de cohorte prospectivo, cuyo objetivo fue evaluar la asociación entre peso corporal (obesidad y no obesidad) y caries dentarias en niños norteamericanos. Se siguió una muestra de 135 niños con edad promedio de 8 años, por un período de 4 meses, no encontrándose asociación en dicha muestra. Sin embargo, la experiencia de caries tomó en cuenta solamente la presencia de lesiones, no incorporando las restauraciones y las pérdidas por motivo de caries como parte de esta, lo que llevó a los autores a encontrar una baja prevalencia $(2,06 \%)$. Esto finalmente puede haber sesgado el resultado en dirección a la ausencia de correlación. El tamaño muestral reducido asociado a un corto período de seguimiento también se mostró como limitante del estudio.

En el año 2006, Mark y col ${ }^{18}$, exploraron la asociación entre sobrepeso y caries dentarias en niños de Estados Unidos de Norteamérica, a través de un estudio de corte transversal por análisis de datos de la encuesta nacional de salud y nutrición de ese país (NHANES), realizada entre 1999-2002. Se efectuó el análisis en niños entre 2 y 17 años de edad. No se encontró asociación significativa entre IMC por edad y prevalencia de caries dental, tanto en dentadura temporal como en definitiva. Para esta última, se encontró que el sobrepeso podría representar incluso un factor protector, pues estuvo asociado a índices promedios más bajos de COPD. 
Estos hallazgos son consistentes con el estudio realizado por Kopicka-Kedzierawski y col $^{19}$, que consistió en fusionar los resultados de dos encuestas nacionales de salud y nutrición de Estados Unidos de Norteamérica (NHANES III y NHANES 1999-2002). Los análisis se realizaron para dentición primaria y definitiva por separado, no entregando una visión global de la experiencia real de caries de gran parte de la muestra, constituida por niños con dentadura mixta. Se encontró que, en niños de 2 a 5 años, no hubo diferencia significativa en la experiencia de caries entre aquellos clasificados como eutróficos y aquellos con sobrepeso. Respecto a los niños de 6 a 11, los diagnosticados con obesidad presentaron un menor riesgo de caries en comparación con los niños de peso normal, en lo referente a la dentadura primaria $(\mathrm{OR}=0,7$; IC95\% 0,5-0,98) y permanente $(\mathrm{OR}=0,6$; IC95\% 0,4-0,95) en la encuesta NHANES III. En la misma encuesta, los adolescentes de 12 a 18 años con sobrepeso presentaron un menor riesgo de caries en comparación con eutróficos $(\mathrm{OR}=0,5 ; \mathrm{IC} 95 \%$ 0,3-0,9). Para los niños entre 6 a 11 y 12 a 18 años, no se encontraron diferencias significativas en la experiencia de caries entre niños con peso normal, sobrepeso y con obesidad en la encuesta NHANES 1999-2002.

Se sabe que el consumo frecuente de carbohidratos y azúcares refinados están fuertemente asociados a la aparición de lesiones de caries $^{20,21}$, y que tal consumo también se asocia al sobrepeso y obesidad infantil ${ }^{10,22}$. De esta forma, era plausible plantear que los niños con mal nutrición por exceso posiblemente tendrían una mayor probabilidad de tener peores indicadores de salud oral. Sin embargo, esto no se corroboró en el presente estudio. Tal situación deja al descubierto la complejidad de la relación entre la mal nutrición por exceso y la experiencia de caries, la cual pudiera no ser explicada únicamente por el patrón de consumo de azúcares.

Otros estudios encontraron una asociación positiva entre experiencia de caries y estado nutricional, por ejemplo, Marshal y col, en $2007^{8}$, estudiaron la asociación entre caries y obesidad infantil, controlando por efectos de dieta y nivel socioeconómico, en un estudio donde los niños eran parte de una cohorte que investigaba la asociación entre exposición al flúor y salud bucal y ósea (Iowa Fluoride Stu$d y)$. Participaron 423 niños que fueron reclutados al nacer y seguidos por un período aproximado de 11 años. Se encontró que los niños con experiencia positiva de caries pertenecían a niveles socioeconómicos más bajos, tenían padres con menor nivel educacional y mayor frecuencia de consumo de bebidas azucaradas en comparación con aquellos niños que no presentaron caries. Los niños con obesidad también pertenecían a niveles socioeconómicos más bajos en comparación con niños eutróficos. En este estudio, los niños con sobrepeso presentaron mayor riesgo de caries en comparación con eutróficos y obesos $(\mathrm{RR}=3,02$; IC95\% 1,46-6,25). Los autores concluyeron que hay una coexistencia de caries y obesidad en niños de bajo nivel socioeconómico.

Willerhausen y col23, también en 2007, investigaron la asociación entre el IMC y la salud dental en niños de enseñanza básica de Alemania, a través de un estudio de corte transversal. Se examinó a 1290 niños para determinar la frecuencia de caries (considerada como lesión clínicamente detectable o descoloración alrededor de restauraciones) y evaluar el estado nutricional de la población. La prevalencia de piezas libres de caries encontrada fue de $44,7 \%$ en niños de bajo peso, $40,7 \%$ en niños con peso normal, $30,5 \%$ en niños con sobrepeso y $31,7 \%$ en niños con obesidad. Se encontró una asociación significativa entre sobrepeso y frecuencia de caries, tanto en dentición primaria como en permanente, aún ajustando por edad y sexo.

Se han publicado otros estudios que plantean la existencia de asociación entre experiencia de caries y estado nutricional en población infantil y adolescente. Sin embargo, debido a su reducido tamaño muestral ${ }^{24,25}$ los hallazgos por ellos reportados no son considerados como válidos.

Las limitaciones del presente estudio recaen en que los datos analizados, por corresponder a un diseño de corte transversal, no entregan evidencias de causalidad. Los examinadores fueron previamente capacitados para la obtención de las variables, sin embargo, no 
se realizó formalmente un análisis de concordancia entre e intra-observadores (Kappa).

Además la higiene dental, la exposición al flúor tópico y la frecuencia del consumo de azúcares no fueron medidas en este trabajo, siendo que estas variables pueden estar distorsionando los hallazgos encontrados. Por otro lado, según datos del Ministerio de Salud de Chile (información no publicada), todos los colegios participantes pertenecen a comunas que cuentan con flúor en dosis óptimas en el agua de abastecimiento (alrededor de 0,6 ppm). No se indagó en la causal de ausencia de los escolares que no asistieron a los establecimientos el día de la evaluación.

Este estudio se focalizó a un segmento socioeconómico específico de la población, por lo que no se buscó encontrar diferencias según esta variable en esta muestra. Debido a que los niños evaluados pertenecen a los colegios de la SIP, es plausible plantear que esta población sería no representativa del estrato socioeconómico medio bajo y bajo de la ciudad de Santiago. Pero, dado que estos establecimientos están distribuidos en distintos puntos geográficos, y considerando que estos planteles no cuentan con criterios de exclusión para el ingreso de los escolares, se podría considerar que este sesgo es menor.

Tales limitaciones plantean la necesidad de realizar nuevas investigaciones, especialmente de carácter prospectivo, donde será interesante evaluar el impacto de las variables no medidas sobre la experiencia de caries en niños con malnutrición por exceso, especialmente del consumo de azúcar y carbohidratos refinados, por relacionarse directamente con la incidencia de caries y también con el incremento del peso corporal.

La prevalencia de caries encontrada en esta población es mayor a la reportada en investigaciones realizadas a nivel nacional, revelando que este segmento de la población de la ciudad de Santiago se encuentra en una condición de especial susceptibilidad en relación a esta patología. Tomando en consideración estos resultados y aquellos reportados por la OPS, se concluye que la situación de la salud oral es comparable a la situación de la mayoría de los países americanos.

\section{Conclusión}

Los hallazgos de este estudio, los cuales concuerdan con los trabajos de mayor representación poblacional y de mejor calidad metodológica, nos permiten concluir que la mal nutrición por exceso no se asocia a la prevalencia de caries en escolares. Estos resultados apuntan a que las medidas y decisiones de políticas públicas relacionadas a combatir la alta prevalencia de caries no debieran pasar por el control del sobrepeso y obesidad infantil, por lo que se debiera seguir poniendo énfasis en educar a los padres y niños acerca de la importancia de la higiene oral, el rol de los azúcares y carbohidratos refinados, el papel del flúor, así como también en el fomento de aquellas intervenciones contempladas en el plan Auge y que están dirigidas a la prevención de esta importante patología.

\section{Referencias}

1.- Kosti RI, Panagiotakos DB: The epidemic of obesity in children and adolescents in the world. Cent Eur J Public Health 2006; 14: 151-9.

2.- Muzzo R, Burrows J, Cordero I, Ramírez I: Trends in nutritional status and stature among school-age children in Chile. Nutrition 2004; 20: 867-72.

3.- Junta Nacional de Auxilio Escolar y Becas: Situación nutricional de los escolares chilenos de primer básico. JUNAEB, 2006. http://sistemas.junaeb.cl/estadosnutricionales_2007/index2.php. Fecha de Acceso: 16/07/08.

4.- World Health Organization: Oral Health http://www. who.int/mediacentre/factsheets/fs318/es/index.html. Fecha de Acceso: 12/09/2008.

5.- MINSAL, Soto L, Tapia R, et al: Diagnóstico nacional de salud bucal de los niños de 6 años. Chile, 2007. http:// www.redsalud.gov.cl/archivos/salud_bucal/perfilepidemiologico.pdf. Fecha de Acceso: 23/06/2008.

6.- Soto L, Tapia $R$, et al: Diagnóstico nacional de salud bucal del adolescente de 12 años y evaluación del grado de cumplimiento de los objetivos sanitarios de salud bucal 2000-2010. Chile 2007. http://www.redsalud.gov. cl/archivos/salud_bucal/perfilepidemiologico.pdf. Fecha de Acceso: 23/06/2008.

7.- Kantovitz KR, Pascon FM, Rontani RM, Gavião MB: Obesity and dental caries: A systematic review. Oral Health Prev Dent 2006; 4: 137-44. 
8.- Marshall TA, Eichenberger-Gilmore JM, Broffit BA, et al: Dental caries and childhood obesity: roles of diet and socioeconomic status. Community Oral Dent Epidemiol 2007; 35: 449-58.

9.- Pinto A, Kim S, Wadenya $R$, et al: Is there association between weight and dental caries among pediatric patients in a urban dental school? A correlation study. J Dent Educ 2007; 71: 1435-40.

10.- Palmer CA: Dental caries and obesity in children: different problems, related causes. Quintessence International 2005; 36: 457-61.

11.- Ludwig DS, Peterson KE, Gortmaker SL: Relation between consumption of sugar-sweetened drinks and childhood obesity: A prospective, observational analysis. Lancet 2001; 357: 505-508.

12.- Random.org. http://www.random.org/ Fecha de Acceso: 29/3/2007.

13.- Kuczmarski RJ, Odgen CL, Grummer-Strawn LM, et al: CDC growth charts: United States. Adv Data 2000; 8: $1-27$.

14.- World Health Organization: Calibration of examiners for oral health epidemiological surveys. Ginebra. WHO, 1987.

15.- World Health Organization: Oral health surveys: basic methods. 3rd. ed. Ginebra. WHO, 1987.

16.- Instituto Nacional de Estadisticas/Comisión Económica para América Latina y el Caribe (CEPAL) Chile: proyecciones y estimaciones de población. Total país 1950-2050. http://www.ine.cl/canales/chile_estadistica/ demografia_y_vitales. Fecha de Acceso: 05/11/2008.
17.- Organización Panamericana de la Salud: 138 Sesión del Comité Ejecutivo PAHO 2006 - Ginebra. www. paho.org/spanish/gov/ce/ee138-14-s.pdf. Fecha de Acceso: $5 / 11 / 2008$.

18.- Mark D, Macek MD, Mitola DJ: Exploring the association between overweight and dental caries among US children. Pediatric Dentistry 2006; 28: 375-9.

19.- Kopycka-Kedzierawski DT, Auinger P, Weitzman M, et al: Caries status in 2-to 18-year-old US children: findings from national surveys. Community Dent Oral Epidemiol 2008; 36: 157-67.

20.- Sheiham A: Dietary effects on dental diseases. Public Health Nutrition 2001; 4: 569-91.

21.- Moyniham P, Petersen PE: Diet, nutrition and the prevention of dental diseases. Public Health Nutrition. 2004; 7 (1A): 201-26.

22.- Malik VS, Schulze MB, Hu FB: Intake of sugar-sweetened beverages and weight gain: a systematic review. Am J Clin Nutr 2006; 84: 274-88.

23.- Willerhausen B, Blettner M, Kasaj A, et al: Association between body mass index and dental health in 1290 children of elementary schools in a german city. Clin Oral Invest 2007; 11: 195-200.

24.- Bailleul-Forestier I, Lopes K, Souames M, et al: Caries experience in a severely obese adolescent population. Int J Paediatr Dent 2007; 17: 358-63.

25.- Hilgers KH, Kinane DF, Scheets JP: Association between childhood obesity and smooth-surface caries in posterior teeth: a preliminary study. Pediatr Dent 2006; 28: 23-7. 